\title{
DNA Binding Protein
}

National Cancer Institute

\section{Source}

National Cancer Institute. DNA Binding Protein. NCI Thesaurus. Code C16518.

Proteins that non-covalently interact with deoxyribonucleic acid as a result of intermolecular physical forces and spatial complementarity. Many genetic regulatory DNA binding proteins bind to double- or single-stranded DNA in a sequence-specific manner and share common structural motifs such as the zinc-finger, helix-turn-helix, and leucine zipper. 